\title{
Spectral Homotopy Analysis Method for PDEs That Model the Unsteady Von Kàrmàn Swirling Flow
}

\author{
Z. G. Makukula and S. S. Motsa ${ }^{\dagger}$ \\ School of Mathematics, Statistics and Computer Sciences, University of KwaZulu-Natal, Private Bag X01, \\ Scottsville 3209, Pietermaritzburg, South Africa \\ †Corresponding Author Email: sandilemotsa@gmail.com
}

(Received November 5, 2013; accepted January 12, 2014)

\begin{abstract}
A spectral homotopy analysis method (SHAM) is used to find numerical solutions for the unsteady viscous flow problem due to an infinite rotating disk. The problem is governed by a set of two fully coupled nonlinear partial differential equations. The method was originally introduced for solutions of nonlinear ordinary differential equations. In this study, its application is extended to a system of nonlinear partial differential equations (PDEs) that model the unsteady von Kàrmàn swirling flow. Numerical values of the pertinent flow properties were generated and validated against results obtained using the Keller-box numerical scheme. The results indicate that the present method is very accurate and can be used as an efficient tool for solving nonlinear PDEs of the type discussed in this paper.
\end{abstract}

Keywords: Swirling flows, Von Kàrmàn flows, Spectral homotopy analysis method, Series solution.

\section{INTRODUCTION}

The study of the steady, laminar and axially symmetric viscous flow due to an infinite disk rotating steadily with constant angular velocity was pioneered by von Kàrmàn (1921). He provided the basis for the mathematical study of the rotating disk problem and introduced his famous transformations that reduced the governing partial differential equations to ordinary differential equations. His solution was improved by Cochran (1934) who patched together two series expansions. Since then solutions of the von Kàrmàn equations describing different aspects of the disk flow problem have been of interest to researchers in diverse fields of science. Swirling flows have many interesting features and occur frequently both in nature and in technology Zandbergen and Dijkstra (1987). Technical applications can be found in viscometry, in lubrication, in centrifuges, in turbines and in the fabrication of computer memories by crystal growth processes (Zandbergen and Dijkstra 1987; Turkyilmazoglu 2011; Sahoo 2009).

The von Kàrmàn swirling viscous flow problem is a well-documented classical problem in fluid mechanics and has been investigated by many researchers experimentally (Dernoncourt et al. 1998; Urzay et al. 2011; Poncet et al. 2007; Nayagam et al. 2009), numerically (Attia 2009; Bouffanais and Jacono 2009; Chawla et al. 2006;
Devi and Devi 2011; Dong et al. 2008) and analytically (Turkyilmazoglu 2011; Abdou 2010; El-Nahhas 2007; Turkyilmazoglu 2010; Öztekin et al. 2002).

In this study we carry out a numerical investigation to test the accuracy and applicability of the spectral homotopy analysis method (SHAM) in solving nonlinear partial differential equations. We consider the problem of unsteady von Kàrmàn swirling viscous flow due to an infinite rotating disk, governed by a set of two fully coupled nonlinear partial differential equations which are derived directly from the exact Navier-Stokes equations. The problem under investigation was previously considered by $\mathrm{Xu}$ and Liao (2006) using the analytic homotopy analysis method (HAM). The proposed SHAM approach is a discrete version of the well-known homotopy analysis method described comprehensively in (Liao 2003; Liao 2012). Some examples of the HAM applications in fluid mechanics related problems can be found in (Rashidi et al. 2008, 2011). The corresponding SHAM was introduced in (Motsa et al. 2010) who used Chebyshev spectral collocation methods to solve the highorder deformation equations in the frame of the HAM. The initial approximation was also found systematically as the solution of the nonhomogeneous linear part of the differential equation to be solved even if it was impossible to solve exactly. The immediate benefit of these innovations 
was better accuracy and faster convergence of the solution series, requiring fewer iterations and less computational effort. Other benefits of the SHAM are that (i) the range of admissible convergence controlling values is much wider in the spectral homotopy analysis method than in the original homotopy analysis method, (ii) the method allows for a much wider range of linear and nonlinear operators.

The use of the spectral homotopy analysis method in fluid mechanics applications has largely been restricted to the solution of systems defined as nonlinear nonlinear ordinary differential equations (Makukula et al. 2010a, 2010b, 2012; Sibanda et al. 2012). An attempt to extend the application of the SHAM to nonlinear partial differential equations was recently presented in Motsa (2013) in which the problem of unsteady boundary layer flow caused by an impulsively stretching plate was investigated. The problem considered in Motsa (2013) was a one equation nonlinear PDE. The aim of this work is to investigate the applicability of the SHAM on the coupled system of nonlinear PDEs considered in Xu and Liao (2006). The accuracy of the SHAM numerical results was validated against results reported in the literature and against those of the Keller-box numerical scheme. The Keller-box method is a popular implicit finite differences based method that has been used by many researchers to solve non-linear differential equations with fluid mechanics applications (see, for example, Molla et al. 2012; Rashad et al. 2013; Prasad et al. 2013).

\section{PROBLEM STATEMENT}

We consider the unsteady, laminar, axially symmetric viscous flow of an incompressible fluid introduced by an infinite disk $(z=0)$ which is started impulsively (at $t=0$ ) into a steady rotation with constant angular velocity $\Omega(\Omega \neq 0)$. The flow satisfies the continuity and Navier- Stokes equations, $\mathrm{Xu}$ and Liao (2006).

$$
\begin{aligned}
& \frac{\partial u}{\partial r}+\frac{u}{r}+\frac{\partial w}{\partial z}=0 \\
& \frac{\partial u}{\partial t}+u \frac{\partial u}{\partial r}+w \frac{\partial u}{\partial z}-\frac{v^{2}}{r}=-\frac{1}{\rho} \frac{\partial p}{\partial r} \\
& +v\left(\frac{\partial^{2} u}{\partial r^{2}}+\frac{1}{r} \frac{\partial u}{\partial r}-\frac{u}{r^{2}}+\frac{\partial^{2} u}{\partial z^{2}}\right) \\
& \frac{\partial v}{\partial t}+u \frac{\partial v}{\partial r}+w \frac{\partial v}{\partial z}+\frac{u v}{r}= \\
& v\left(\frac{\partial^{2} v}{\partial r^{2}}+\frac{1}{r} \frac{\partial v}{\partial r}-\frac{v}{r^{2}}+\frac{\partial^{2} v}{\partial z^{2}}\right) \\
& \frac{\partial w}{\partial t}+u \frac{\partial w}{\partial r}+w \frac{\partial w}{\partial z}=-\frac{1}{\rho} \frac{\partial p}{\partial z} \\
& +v\left(\frac{\partial^{2} w}{\partial r^{2}}+\frac{1}{r} \frac{\partial w}{\partial r}+\frac{\partial^{2} w}{\partial z^{2}}\right)
\end{aligned}
$$

subject to the initial and boundary conditions

$$
\begin{aligned}
& u=v=w=0, \quad \text { at } \quad t=0, \\
& u=w=0, \quad v=r \Omega, \quad \text { at } \quad z=0, \\
& u=v=0, \quad \text { as } \quad z \rightarrow \infty,
\end{aligned}
$$

where $u, v, w$ are velocity components in the directions of increasing $r, \varphi$ and $z, t$ denotes the time, $p$ the pressure, $v$ the coefficient of kinematic viscosity and $\rho$ the density of the fluid, respectively. Following Xu and Liao (2006), we use the similarity variables

$\eta=z \sqrt{\frac{\Omega}{v \xi}}, \quad \xi=1-e^{\tau}, \quad \tau=\Omega t$,

(6)

and similarity transformations

$u=r \Omega f(\eta, \xi), \quad v=r \Omega g(\eta, \xi)$,

$w=\sqrt{r \Omega \xi} s(\eta, \xi), \quad p=-\rho v \Omega P(\eta, \xi)$.

The governing equations are reduced to

$\frac{\partial^{3} s}{\partial \eta^{3}}+(1-\xi)\left(\frac{\eta}{2} \frac{\partial^{2} s}{\partial \eta^{2}}-\xi \frac{\partial^{2} s}{\partial \xi \partial \eta}\right)$

$+\xi\left\{\frac{1}{2}\left(\frac{\partial s}{\partial \eta}\right)^{2}-s \frac{\partial^{2} s}{\partial \eta^{2}}-2 g^{2}\right\}=0$,

$\frac{\partial^{2} g}{\partial \eta^{2}}-(1-\xi)\left(\xi \frac{\partial g}{\partial \xi}-\frac{\eta}{2} \frac{\partial g}{\partial \xi}\right)$

$+\xi\left(g \frac{\partial s}{\partial \eta}-s \frac{\partial g}{\partial \eta}\right)=0$

subject to the boundary conditions

$g(0, \xi)=1, \quad s(0, \xi)=0,\left.\quad \frac{\partial s(\eta, \xi)}{\partial \eta}\right|_{\eta=0}=0$,

$g(\infty, \xi)=0,\left.\quad \frac{\partial s(\eta, \xi)}{\partial \eta}\right|_{\eta=+\infty}=0$.

(10)

The skin friction coefficients in the radial and tangential directions are given by, $\mathrm{Xu}$ and Liao (2006)

$C_{f}^{r}=\frac{\tau_{r}}{\rho(r \Omega)^{2}}=-\frac{f^{\prime}(0, \xi)}{\sqrt{\xi R e_{r}}}=\frac{s^{\prime \prime}(0, \xi)}{2 \sqrt{\xi R e_{r}}}$,

(11)

$C_{f}^{\varphi}=\frac{\tau_{\varphi}}{\rho(r \Omega)^{2}}=-\frac{g^{\prime}(0, \xi)}{\sqrt{\xi R e_{r}}}$

where $R e_{r}=r \sqrt{\Omega / v}$ represents the local Reynolds number, $\tau_{r}$ and $\tau_{\varphi}$ are the radial and tangential shear stress, respectively.

\section{METHOD OF SOLUTION}

The spectral homotopy analysis method (SHAM) is a discrete version of the homotopy analysis method (HAM) which is employed to decompose a system of nonlinear differential equations into a sequence of linear ordinary differential equations. In the discrete version of the HAM, the linearised equations are solved using the Chebyshev spectral collocation method. Spectral methods are preferred over other numerical solutions for problems with smooth solutions because of their pronounced accuracy (Canuto et al. 2007; Hesthaven et al. 2007).

In the framework of the SHAM, the nonlinear equations are decomposed into their linear and nonlinear parts and the governing Eqs. (9) and (10) are written, respectively, as follows;

$L_{s}[s(\eta, \xi)]+N_{s}[s(\eta, \xi), g(\eta, \xi)]=0$,

$L_{g}[g(\eta, \xi)]+N_{g}[s(\eta, \xi), g(\eta, \xi)]=0$,

where

$$
\begin{aligned}
& L_{s}[s]=\frac{\partial^{3} s}{\partial \eta^{3}}+(1-\xi)\left(\frac{\eta}{2} \frac{\partial^{2} s}{\partial \eta^{2}}-\xi \frac{\partial^{2} s}{\partial \xi \partial \eta}\right), \\
& N_{s}[s, g]=\xi\left\{\frac{1}{2}\left(\frac{\partial s}{\partial \eta}\right)^{2}-s \frac{\partial^{2} s}{\partial \eta^{2}}-2 g^{2}\right\}
\end{aligned}
$$




$$
\begin{aligned}
& L_{g}[g]=\frac{\partial^{2} g}{\partial \eta^{2}}-(1-\xi)\left(\xi \frac{\partial g}{\partial \xi}-\frac{\eta}{2} \frac{\partial g}{\partial \xi}\right), \\
& N_{g}[s, g]=\xi\left(g \frac{\partial s}{\partial \eta}-s \frac{\partial g}{\partial \eta}\right),
\end{aligned}
$$

with $L_{s}, L_{g}$ and $N_{s}, N_{g}$ representing the linear and nonlinear operators of the equations respectively We note that the linear operators used in (15) and (17) are PDEs with variable coefficients. This is in direct contrast to the standard linear operator that was used in (Xu and Liao 2006) which was defined as the linear operators

$$
\begin{aligned}
& \mathcal{L}_{S}(S)=\frac{\partial^{3} S}{\partial \eta}-\frac{\partial S}{\partial \eta}, \\
& \mathcal{L}_{g}(G)=\frac{\partial^{2} G}{\partial \eta}-\frac{\partial G}{\partial \eta} .
\end{aligned}
$$

The HAM formulation starts with the construction of the zeroth-order deformation equations are formulated as;

$(1-q) L_{s}\left[S(\eta, \xi ; q)-s_{0}(\eta, \xi)\right]=$ $q \hbar\left\{L_{s}[S(\eta, \xi ; q)]+N_{S}[S(\eta, \xi ; q), G(\eta, \xi ; q)]\right\}$, $(1-q) L_{g}\left[G(\eta, \xi ; q)-g_{0}(\eta, \xi)\right]=$ $q \hbar\left\{L_{g}[S(\eta, \xi ; q)]+N_{g}[S(\eta, \xi ; q), G(\eta, \xi ; q)]\right\},(21)$

subject to the boundary conditions $G(0, \xi ; q)=1, \quad S(0, \xi ; q)=0$,

$\left.\frac{\partial S(\eta, \xi ; q)}{\partial \eta}\right|_{\eta=0}=0, G(\infty, \xi ; q)=0$,

$\left.\frac{\partial S(\eta, \xi ; q)}{\partial \eta}\right|_{\eta=+\infty}=0$,

where $q \in[0,1]$ is an embedding parameter and $\hbar$ is the convergence controlling parameters. The functions $S(\eta, \xi ; q)$ and $G(\eta, \xi ; q)$ are unknown functions and $s_{0}(\eta, \xi)$ and $g_{0}(\eta, \xi)$ are the initial approximate solutions. Higher order deformation equations are derived by differentiating the zerothorder deformation Eqs. (20) and (21) $m$ times with respect to $q$ then dividing by $m$ ! to get

$$
\begin{gathered}
L_{s}\left[s_{m}(\eta, \xi)-\left(\chi_{m}+\hbar\right) s_{m-1}(\eta, \xi)\right]=\hbar R_{m}^{s}(\eta, \xi), \\
L_{g}\left[g_{m}(\eta, \xi)-\left(\chi_{m}+\hbar\right) g_{m-1}(\eta, \xi)\right]=\hbar R_{m}^{g}(\eta, \xi),
\end{gathered}
$$

with

$$
\begin{gathered}
R_{m}^{S}(\eta, \xi)=\left.\frac{1}{(m-1) !} \frac{\partial^{m-1}}{\partial q^{m-1}}\left\{L_{s}+N_{s}\right\}\right|_{q=0}, \\
R_{m}^{g}(\eta, \xi)=\left.\frac{1}{(m-1) !} \frac{\partial^{m-1}}{\partial q^{m-1}}\left\{L_{g}+N_{g}\right\}\right|_{q=0},
\end{gathered}
$$

and

$$
\chi_{m}= \begin{cases}0, & m \leq 1 \\ 1, & m>1\end{cases}
$$

The higher order deformation Eqs. (23) and (24) can be explicitly written as

$$
\begin{aligned}
& \mathcal{L}_{s}\left[s_{m}(\eta, \xi)\right]=\left(\hbar+\chi_{m}\right) \mathcal{L}_{s}\left[s_{m-1}(\eta, \xi)\right] \\
& +\hbar \xi \sum_{n=0}^{m-1}\left[\frac{1}{2} s^{\prime}{ }_{m-1-n} s^{\prime}{ }_{n}-s_{m-1-n} s^{\prime \prime}{ }_{n}-\right. \\
& \left.2 g_{m-1-n} g_{n}\right], \\
& \mathcal{L}_{g}\left[g_{m}(\eta, \xi)\right]=\left(\hbar+\chi_{m}\right) \mathcal{L}_{g}\left[g_{m-1}(\eta, \xi)\right] \\
& +\hbar \xi \sum_{n=0}^{m-1}\left[g_{m-1-n} s^{\prime}{ }_{n}-s_{m-1-n} g^{\prime}{ }_{n}\right], \ldots \ldots .
\end{aligned}
$$$$
\text { respectively subject to the boundary conditions }
$$

$$
\begin{gathered}
g_{m}(0, \xi)=1, \quad s_{m}(0, \xi)=0,\left.\quad \frac{\partial s_{m}(\eta, \xi)}{\partial \eta}\right|_{\eta=0} \\
=0, \\
g_{m}(\infty, \xi)=0,\left.\quad \frac{\partial s_{m}(\eta, \xi)}{\partial \eta}\right|_{\eta=+\infty}=0 \ldots \ldots
\end{gathered}
$$

In their form, Eqs. (27) and (28) are difficult to solve using the traditional HAM approach of $\mathrm{Xu}$ and Liao (2006). However, if we assume a series expansion in the $\xi$-direction, the problem can be dealt with quite easily in the frame of the SHAM. To this end, we assume that the solution can be expressed in the form

$s(\eta, \xi)=\sum_{m=0}^{+\infty} \sum_{k=0}^{+\infty} \xi^{k} s_{m, k}(\eta)$,

with $s_{m}(\eta, \xi)=\sum_{k=0}^{+\infty} \xi^{k} s_{m, k}(\eta, \xi)$,

$g(\eta, \xi)=\sum_{m=0}^{+\infty} \sum_{k=0}^{+\infty} \xi^{k} g_{m, k}(\eta)$,

with $g_{m}(\eta, \xi)=\sum_{k=0}^{+\infty} \xi^{k} g_{m, k}(\eta, \xi)$.

The initial solutions are chosen to obey the rule of solution expressions (30) and (31) respectively and satisfying the boundary conditions of the governing Eq. (29) such that

$$
\begin{aligned}
& \mathcal{L}_{s}\left[s_{0}(\eta, \xi)\right]=0, \quad s_{0}(0, \xi)=0, \\
& s^{\prime}{ }_{0}(0, \xi)=0, \quad s^{\prime}{ }_{0}(\infty, \xi)=0, \\
& \mathcal{L}_{g}\left[g_{0}(\eta, \xi)\right]=0, \quad g_{0}(0, \xi)=1, \\
& g_{0}(\infty, \xi)=0
\end{aligned}
$$

Substituting Eq. (30) and (31) into Eqs. (32) and (33) respectively, and balancing terms of equal order in $\xi$ gives

$$
\begin{aligned}
& s^{\prime \prime \prime}{ }_{0,0}+\frac{\eta}{2} s^{\prime \prime}{ }_{0,0}=0 \\
& g^{\prime \prime}{ }_{0,0}+\frac{\eta}{2} g^{\prime}{ }_{0,0}=0 \\
& s_{0,0}(0)=s^{\prime}{ }_{0,0}(0)=s^{\prime}{ }_{0,0}(\infty)=0, \\
& g_{0,0}(0)=1, \quad g_{0,0}(\infty)=0 .
\end{aligned}
$$

For $k \geq 1$ the resulting equations are

$s^{\prime \prime \prime}{ }_{0, k}+\frac{\eta}{2} s^{\prime \prime}{ }_{0, k}-k s^{\prime}{ }_{0, k}$

$=\frac{\eta}{2} s^{\prime \prime}{ }_{0, k-1}-(k-1) s^{\prime}{ }_{0, k-1}$,

$s_{0, k}(0)=s^{\prime}{ }_{0, k}(0)=s^{\prime}{ }_{0, k}(\infty)=0$,

$g^{\prime \prime}{ }_{0, k}+\frac{\eta}{2} g_{0, k}^{\prime}-k g_{0, k}=\frac{\eta}{2} g_{0, k-1}^{\prime}$

$-(k-1) g_{0, k-1}$

$g_{0, k}(0)=g_{0, k}(\infty)=0$.

Substituting Eqs. (30) and (31) into the higher order deformation Eqs. (27) and (28) respectively and balancing terms of order $\xi$ gives rise to the following equations when $k=0$,

$$
\begin{aligned}
s^{\prime \prime \prime}{ }_{m, 0}+\frac{\eta}{2} s^{\prime \prime}{ }_{m, 0}= & \left(\hbar+\chi_{m}\right)\left\{s^{\prime \prime \prime}{ }_{m-1,0}\right. \\
& \left.+\frac{\eta}{2} s^{\prime \prime}{ }_{m-1,0}\right\}, \\
s_{m, 0}(0)=s^{\prime}{ }_{m, 0}(0) & =s^{\prime}{ }_{m, 0}(\infty)=0, \\
g^{\prime \prime}{ }_{m, 0}+\frac{\eta}{2} g^{\prime}{ }_{m, 0}= & \left(\hbar+\chi_{m}\right)\left\{g^{\prime \prime}{ }_{m-1,0}\right. \\
& \left.+\frac{\eta}{2} g^{\prime}{ }_{m-1,0}\right\}, \\
g_{m, 0}(0)=g_{m, 0}(\infty) & =0 .
\end{aligned}
$$


The higher order deformation equations when $m \geq 1$, are

$$
\begin{aligned}
& s^{\prime \prime \prime}{ }_{m, k}+\frac{\eta}{2} s^{\prime \prime}{ }_{m, k}-k s^{\prime}{ }_{m, k}= \\
& \left(\hbar+\chi_{m}\right)\left(s^{\prime \prime \prime}{ }_{m-1, k}+\frac{\eta}{2} s^{\prime \prime}{ }_{m-1, k}-k s^{\prime}{ }_{m-1, k}\right) \\
& +\left(\hbar+\chi_{m}\right)\left((k-1) s^{\prime}{ }_{m-1, k-1}-\frac{\eta}{2} s^{\prime \prime}{ }_{m-1, k-1}\right) \\
& +\frac{\eta}{2} s^{\prime \prime}{ }_{m, k-1}-(k-1) s^{\prime}{ }_{m, k-1} \\
& +\hbar \sum_{n=0}^{m-1} \sum_{p=0}^{k-1}\left[\frac{1}{2} s^{\prime}{ }_{m-1-n, k-1-p} s^{\prime}{ }_{n, p}\right. \\
& \left.-s_{m-1-n, k-1-p} s^{\prime \prime}{ }_{n, p}-2 g_{m-1-n, k-1-p} g_{n, p}\right], \\
& g^{\prime \prime}{ }_{m, k}+\frac{\eta}{2} g^{\prime}{ }_{m, k}-k g_{m, k}= \\
& \left(\hbar+\chi_{m}\right)\left(g^{\prime \prime}{ }_{m-1, k}+\frac{\eta}{2} g^{\prime}{ }_{m-1, k}-k g_{m-1, k}\right) \\
& +\left(\hbar+\chi_{m}\right)\left((k-1) g_{m-1, k-1}-\frac{\eta}{2} g^{\prime}{ }_{m-1, k-1}\right) \\
& +\frac{\eta}{2} g^{\prime}{ }_{m, k-1}-(k-1) g_{m, k-1} \\
& +\hbar \sum_{n=0}^{m-1} \sum_{p=0}^{k-1}\left[s_{m-1-n, k-1-p} g_{n, p}\right. \\
& \left.-s_{m-1-n, k-1-p} g^{\prime}{ }_{n, p}\right],
\end{aligned}
$$

subject to the boundary conditions

$$
\begin{aligned}
& g_{m, k}(0)=0, s_{m, k}(0)=0, s^{\prime}{ }_{m, k}(0)=0 \\
& g_{m, k}(\infty)=0, s^{\prime}{ }_{m, k}(\infty)=0 .
\end{aligned}
$$

Since the right hand side of Eqs. (40) and (41) for $k \geq 1$ are known from previous iterations, the equations may be solved using any numerical method. In this work, we apply the Chebyshev spectral collocation method to integrate Eqs. (40) and (41). The method is based on the Chebyshev polynomials defined on the interval $[-1,1]$ by

$$
T_{i}(x)=\cos \left[i \cos ^{-1}(x)\right] \text {. }
$$

We first transform the physical region $[0, \infty)$ into the region $[-1,1]$ using the domain truncation technique. The problem is solved in the interval $\left[0, \eta_{\infty}\right]$ instead of $[0, \infty)$. This leads to the following algebraic mapping

$x=\frac{2 \eta}{\eta_{\infty}}-1, \quad x \in[-1,1]$

where $\eta_{\infty}$ is the scaling parameter used to invoke the boundary condition at infinity. The Chebyshev nodes in $[-1,1]$ are defined by the Gauss-Lobatto collocation points (Canuto et al. 1988; Trefethen 2000) given by

$x_{j}=\cos \frac{\pi j}{N}, \quad x \in[-1,1]$

for $j=0,1, \ldots, N$ where $N+1$ is the number of collocation points. The unknown functions $s_{m, k}$ and $g_{m, k}$ are approximated using a Lagrange form of interpolating polynomial which interpolates $s_{m, k}$ and $g_{m, k}$ at the Gauss-Lobatto collocation points (45). This gives vector functions $\boldsymbol{S}_{m, k}$ and $\boldsymbol{G}_{m, k}$ which are associated with the functions $s_{m, k}$ and $g_{m, k}$, respectively, at the collocation points. The derivatives are obtained by differentiating the interpolating functions at the collocation points to give

$$
\begin{aligned}
& \left.\frac{d^{r} s_{m, k}}{d x^{r}}\right|_{x=x_{i}}=\sum_{i=0}^{N} \boldsymbol{D}_{i j}^{r} s_{m, k}\left(x_{j}\right)=\boldsymbol{D}^{r} \boldsymbol{S}_{m, k} \\
& \left.\frac{d^{r} g_{m, k}}{d x^{r}}\right|_{x=x_{i}}=\sum_{i=0}^{N} \boldsymbol{D}_{i j}^{r} g_{m, k}\left(x_{j}\right)=\boldsymbol{D}^{r} \boldsymbol{G}_{m, k}
\end{aligned}
$$

where $r$ is the order of differentiation, $\boldsymbol{D}=\frac{2}{\eta_{\infty}} \mathcal{D}$ and $\mathcal{D}$ is the Chebyshev spectral differentiation matrix whose entries are given by

$\mathcal{D}_{i j}= \begin{cases}\frac{c_{j}}{c_{i}} \frac{(-1)^{j+i}}{x_{j}-x_{i}}, & j \neq i, \\ -\frac{x_{i}}{2\left(1-x_{i}^{2}\right)}, & 1 \leq j=i \leq N-1, \\ \frac{2 N^{2}+1}{6}, & j=i=0, \\ -\frac{2 N^{2}+1}{6}, & j=i=N,\end{cases}$

where

$$
c_{j}= \begin{cases}2, & j=0, N \\ 1, & 1,2, \ldots, N-1 .\end{cases}
$$

Substituting Eqs. (44) to (46) in (40) to (42) yields

$\boldsymbol{A}_{1} \boldsymbol{S}_{m, k}=\boldsymbol{B}_{1}, \quad \sum_{i=0}^{N} \boldsymbol{D}_{0 i} s_{m, k}\left(x_{i}\right)=0$,
$\sum_{i=0}^{N} \boldsymbol{D}_{N i} s_{m, k}\left(x_{i}\right)=0, s_{m, k}\left(x_{N}\right)=0$,

$\boldsymbol{A}_{2} \boldsymbol{G}_{m, k}=\boldsymbol{B}_{2}$

$g_{m, k}\left(x_{0}\right)=0, \quad g_{m, k}\left(x_{N}\right)=0$,

where

$\boldsymbol{S}_{m, k}=\left[s_{m, k}\left(x_{0}\right), s_{m, k}\left(x_{1}\right), \ldots, s_{m, k}\left(x_{N}\right)\right]^{T}$,

$\boldsymbol{G}_{m, k}=\left[g_{m, k}\left(x_{0}\right), g_{m, k}\left(x_{1}\right), \ldots, g_{m, k}\left(x_{N}\right)\right]^{T}$,

$\boldsymbol{A}_{1}=\boldsymbol{D}^{3}+\operatorname{diag}\left(\frac{\eta}{2}\right) \boldsymbol{D}^{2}-k \boldsymbol{D}$,

$\boldsymbol{A}_{2}=\boldsymbol{D}^{2}+\operatorname{diag}\left(\frac{\eta}{2}\right) \boldsymbol{D}-k \boldsymbol{I}$.

In the above definitions $I$ is an $(N+1) \times(N+1)$ identity matrix and $\operatorname{diag}()$ represents a diagonal matrix of size $(N+1) \times(N+1)$. The vectors $\boldsymbol{B}_{1}$ and $\boldsymbol{B}_{2}$ are generated by evaluating the right hand side of Eqs (40) and (41) respectively at the collocation points with the derivatives replaced by spectral differentiation matrices.

Suitable initial approximate solutions are found by solving the initial unsteady state solutions at $\xi=0,(\tau=0)$ of Eqs. (8) and (9) given by

$s^{\prime \prime \prime}(\eta, 0)+\frac{1}{2} \eta s^{\prime \prime}(\eta, 0)=0$,

$s(0,0)=0, s^{\prime}(0,0)=0, s^{\prime}(\infty, 0)=0$,

$g^{\prime \prime}(\eta, 0)+\operatorname{Pr} \frac{1}{2} \eta \theta^{\prime}(\eta, 0)=0$

$g(0,0)=1, g(\infty, 0)=0$,

giving

$s_{0,0}=0, \quad g_{0,0}=\operatorname{erfc}(\eta / 2)$,

respectively. The function $\operatorname{erfc}(\eta)$ is the standard complementary error function defined by

$\operatorname{erfc}(\eta)=\frac{2}{\sqrt{\pi}} \int_{\eta}^{\infty} \exp \left(-z^{2}\right) d z$ 
The solutions $s_{m, k}$ and $g_{m, k},(m, k) \geq 1$ can be obtained consequently from

$\boldsymbol{S}_{m, k}=\boldsymbol{A}_{1}^{-1} \boldsymbol{B}_{1}, \quad$ and $\quad \boldsymbol{G}_{m, k}=\boldsymbol{A}_{2}^{-1} \boldsymbol{B}_{2}$.

The approximate solutions for $s(\eta, \xi)$ and $g(\eta, \xi)$ are given by the following finite series approximations

$$
\begin{aligned}
& s(\eta, \xi)=\sum_{m=0}^{M} \sum_{k=0}^{K} \xi^{k} s_{m, k}(\eta), \\
& g(\eta, \xi)=\sum_{m=0}^{M} \sum_{k=0}^{K} \xi^{k} g_{m, k}(\eta)
\end{aligned}
$$

\section{RESULTS AND DISCUSSION}

Numerical solutions to the governing system of partial differential Eqs. (9) and (10) were computed using the spectral homotopy analysis method (SHAM). The solution process was started from the initial analytical solutions at $\xi=0$, corresponding to $\tau=0$. The series form of the higher order deformation equations of the SHAM was used to solve the system for solutions up to close to the steady state at $\xi=1$. Simulations were carried out numerically to obtain approximate solutions of the skin friction coefficients in the radial and tangential directions. The results obtained were validated against those of $\mathrm{Xu}$ and Liao (2006) and against numerical results obtained using the Keller-box implicit finite difference method as described by Cebeci and Bradshaw (1984). The Keller-box method is a well-documented numerical scheme known to be accurate, fast and easier to program for boundary layer flow problems. After a series of numerical trials it was discovered that optimal results are obtained for values of $N=100$ and $\eta_{\infty}=30$. The residual error was also used to check the accuracy of the proposed SHAM . Series solutions were generated until the maximum error of the residual was less than $10^{-6}$. The maximum error of the residual was calculated from the equations

$$
\begin{aligned}
& \operatorname{Res}(s)=\max _{j} \mid \overline{\mathcal{N}}_{s}\left[s\left(\eta_{j}, \xi\right), g(\eta, \xi)\right], \\
& \operatorname{Res}(g)=\max _{j} \mid \overline{\mathcal{N}}_{g}\left[s\left(\eta_{j}, \xi\right), g(\eta, \xi)\right], \\
& j=0,1,2, \ldots, N,
\end{aligned}
$$

where

$\overline{\mathcal{N}}_{s}=L_{s}[s(\eta, \xi)]+N_{s}[s(\eta, \xi), g(\eta, \xi)]=0$,

$\overline{\mathcal{N}}_{g}=L_{g}[g(\eta, \xi)]+N_{g}[s(\eta, \xi), g(\eta, \xi)]=0 .(60)$ In the Tables that follow the values of $K$ and $M$ represent the number of iterations it took a certain solution to converge for a specified accuracy.

Figures 1 and 2 present the maximum error of the residual for $s(\eta, \xi)$ and $g(\eta, \xi)$ respectively as functions of the convergence controlling parameter $\hbar$ at different iterations. From the figures we are able to approximate the optimal value of $\hbar$ which corresponds to the smallest possible residual error at each iteration. As can be seen from the figures, increasing the number of iterations decreases the residual error. For example, in Fig. 1, when $K=M=20$ the residual error is less than $10^{-9}$ compared to about $10^{-3}$ for $K=M=5$. The optimal value of $\hbar$ that gives the smallest error is shown in Fig. 1 to be in the range $-1.1 \leq \hbar \leq-0.8$ at all iterations. The plots in Fig. 2 rather show a single optimal value in each case and as seen from the figure it is roughly $\hbar=-1$ when $K=M=$ $5,15,20$ and about $\hbar=-0.9$ for $K=M=10$.

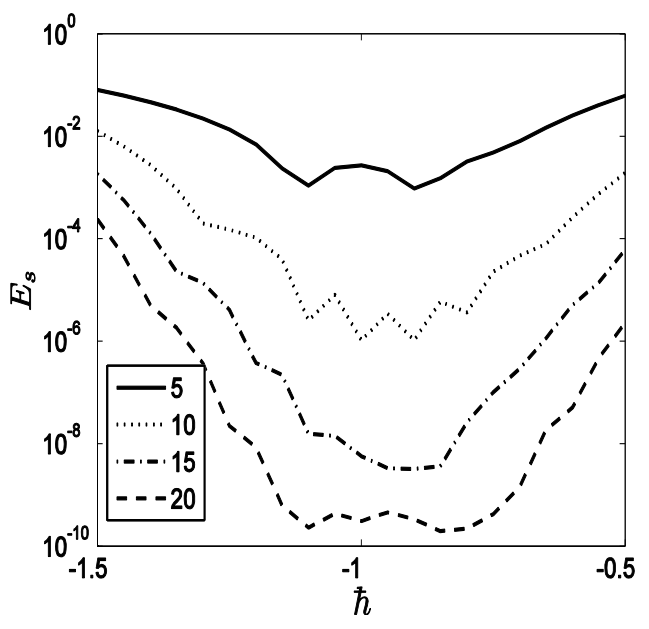

Fig. 1. Maximum residual error for $s(\eta, \xi)$

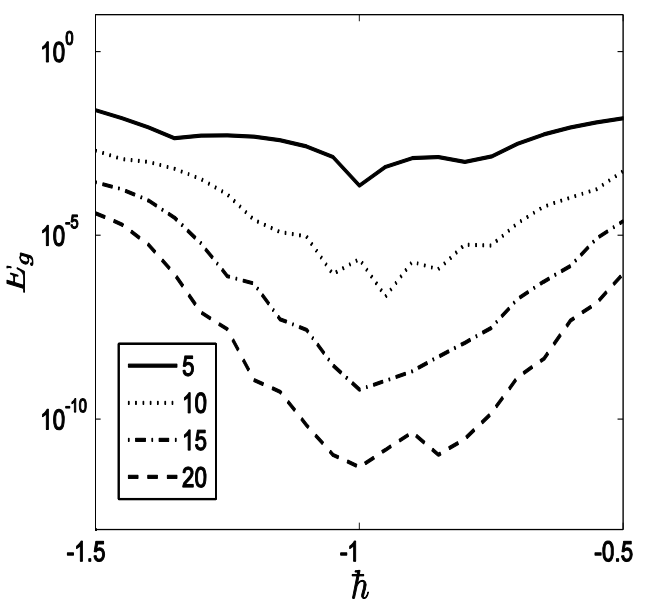

Fig. 2. Maximum residual error for $g(\eta, \xi)$

Table 1 compares the SHAM solutions for the radial skin friction coefficient $\left(s^{\prime \prime}(0, \xi)\right)$ at different values of $\xi$ against those of Xu and Liao (2006) and those of the Keller-box numerical scheme. The SHAM results are in good agreement with those of the Keller-box for all values of $\xi$. Similar trends are observed with the tangential skin friction coefficient values $\left(g^{\prime}(0, \xi)\right)$ in Table 2 and $s(\infty, \xi)$ in Table 3 where exact match with the Keller-box results is observed. The results are also comparable with the results reported in Xu and Liao (2006). We remark here that the Keller-box method used to generate all the tabulated results was implemented with very small step sizes in order to ensure high accuracy. All Tables reveal a decrease in the values of $s^{\prime \prime}(0, \xi), g^{\prime}(0, \xi)$ and $s(\infty, \xi)$ when approaching the steady state solution. 
Z. G. Makukula and S. S. Motsa / JAFM, Vol. 7, No. 4, pp. 711-718, 2014.

Table 1 SHAM solutions for $s^{\prime \prime}(0, \xi)$ against those of $\mathrm{Xu}$ and Liao and the Keller-box at different values of $\xi$ for $M=K=50, \hbar=$ $-1, L=30, N=100$.

\begin{tabular}{|c|c|c|c|c|}
\hline & \multicolumn{2}{|c}{ Xu and Liao (2006) } & \multicolumn{2}{c|}{ Present } \\
\hline$\xi$ & Numerical & HAM & Keller-box & SHAM \\
\hline 0.1 & -0.0841074 & -0.0841062 & -0.084117 & -0.084117 \\
\hline 0.2 & -0.172689 & -0.172688 & -0.172708 & -0.172709 \\
\hline 0.3 & -0.266130 & -0.266129 & -0.266158 & -0.266158 \\
\hline 0.4 & -0.364801 & -0.364799 & -0.364836 & -0.364836 \\
\hline 0.5 & -0.468997 & -0.468996 & -0.469038 & -0.469038 \\
\hline 0.6 & -0.578810 & -0.578809 & -0.578853 & -0.578854 \\
\hline 0.7 & -0.693825 & -0.693824 & -0.693867 & -0.693868 \\
\hline 0.8 & -0.812375 & -0.812374 & -0.812411 & -0.812412 \\
\hline 0.9 & -0.929237 & -0.929239 & -0.929267 & -0.929268 \\
\hline
\end{tabular}

Table 2 SHAM solutions for $g^{\prime}(0, \xi)$ against those of Xu and Liao and the Keller-box at different values of $\xi$ for $M=K=20, \hbar=$ $-1, L=30, N=100$

\begin{tabular}{|c|c|c|c|c|}
\hline & \multicolumn{2}{|c|}{ Xu and Liao (2006) } & \multicolumn{2}{c|}{ Present } \\
\hline$\xi$ & Numerical & HAM & Keller-box & SHAM \\
\hline 0.1 & -0.550693 & -0.550707 & -0.550668 & -0.550668 \\
\hline 0.2 & -0.538573 & -0.538585 & -0.538552 & -0.538552 \\
\hline 0.3 & -0.528308 & -0.528316 & -0.528290 & -0.528290 \\
\hline 0.4 & -0.520502 & -0.520512 & -0.520488 & -0.520488 \\
\hline 0.5 & -0.515989 & -0.515997 & -0.515977 & -0.515977 \\
\hline 0.6 & -0.515940 & -0.515947 & -0.515930 & -0.515930 \\
\hline 0.7 & -0.522060 & -0.522067 & -0.522051 & -0.522051 \\
\hline 0.8 & -0.536944 & -0.536953 & -0.536935 & -0.536935 \\
\hline 0.9 & -0.564858 & -0.564871 & -0.564845 & -0.564845 \\
\hline
\end{tabular}

Table 3 SHAM solutions for $s(\infty, \xi)$ against those of $\mathrm{Xu}$ and Liao and the Keller-box at different values of $\xi$ for $M=K=50, \hbar=$ $-1, L=30, N=100$

\begin{tabular}{|c|c|c|c|c|}
\hline & \multicolumn{2}{|c|}{ Xu and Liao } & \multicolumn{2}{c|}{ Present } \\
\hline$\xi$ & Numerical & HAM & Keller-box & SHAM \\
\hline 0.1 & -0.0361299 & -0.0361355 & -0.036128 & -0.036128 \\
\hline 0.2 & -0.0782994 & -0.0783056 & -0.078294 & -0.078294 \\
\hline 0.3 & -0.127749 & -0.127758 & -0.127738 & -0.127738 \\
\hline 0.4 & -0.186021 & -0.186034 & -0.186003 & -0.186003 \\
\hline 0.5 & -0.255031 & -0.255049 & -0.255005 & -0.255005 \\
\hline
\end{tabular}

\begin{tabular}{|l|l|l|l|l|}
\hline 0.6 & -0.33714 & -0.337165 & -0.337103 & -0.337103 \\
\hline 0.7 & -0.435198 & -0.435202 & -0.435148 & -0.435148 \\
\hline 0.8 & -0.552471 & -0.552517 & -0.552406 & -0.552405 \\
\hline 0.9 & -0.695393 & -0.695411 & -0.692220 & -0.692217 \\
\hline
\end{tabular}

Table $4 s^{\prime \prime}(0, \xi)$ at different values of $\xi$ when

$$
\hbar=-\mathbf{1}
$$

\begin{tabular}{|c|c|c|c|c|}
\hline iter. & $\xi=0.2$ & $\xi=0.4$ & $\xi=0.6$ & $\xi=0.8$ \\
\hline 4 & -0.172706 & -0.364678 & -0.576309 & -0.780669 \\
\hline 6 & -0.172709 & -0.364840 & -0.579076 & -0.820966 \\
\hline 8 & -0.172709 & -0.364836 & -0.578835 & -0.810158 \\
\hline 12 & -0.172709 & -0.364836 & -0.578854 & -0.812266 \\
\hline 16 & -0.172709 & -0.364836 & -0.578854 & -0.812404 \\
\hline 18 & -0.172709 & -0.364836 & -0.578854 & -0.812414 \\
\hline 20 & -0.172709 & -0.364836 & -0.578854 & -0.812412 \\
\hline 22 & -0.172709 & -0.364836 & -0.578854 & -0.812412 \\
\hline 24 & -0.172709 & -0.364836 & -0.578854 & -0.812412 \\
\hline $\begin{array}{c}\text { Keller- } \\
\text { box }\end{array}$ & -0.172708 & -0.364836 & -0.578853 & -0.812411 \\
\hline
\end{tabular}

In order to investigate the speed at which the proposed SHAM converges to the Keller-box numerical results, we present the number of iterations required to reach a certain level of accuracy for $s^{\prime \prime}(0, \xi), g^{\prime}(0, \xi)$, and $s(\infty, \xi)$ in Tables 4 to 6 respectively. For smaller values of $\xi$, convergence of up to five decimal places is reached after six iterations and may take up to fourteen iterations for values of $\xi$ close and equal to one. It can be seen from the tables, that the method converges fully to the numerical results after only a few iterations in all cases. Again the slower convergence rates for $s^{\prime \prime}(0, \xi)$ and $s(\infty, \xi)$ are observed as compared to $g^{\prime}(0, \xi)$. These results indicate that the proposed SHAM approach is a viable solution tool for solving the type of nonlinear PDEs considered in this study.

Table $5 g^{\prime}(0, \xi)$ at different values of $\xi$ when

\begin{tabular}{|c|c|c|c|c|}
\hline iter. & $\xi=0.2$ & $\xi=0.4$ & $\xi=0.6$ & $\xi=0.8$ \\
\hline 2 & -0.538572 & -0.521004 & -0.520549 & -0.569336 \\
\hline 4 & -0.538551 & -0.520474 & -0.515534 & -0.529007 \\
\hline 6 & -0.538552 & -0.520488 & -0.515963 & -0.538558 \\
\hline 8 & -0.538552 & -0.520488 & -0.515928 & -0.536683 \\
\hline 10 & -0.538552 & -0.520488 & -0.515930 & -0.536956 \\
\hline 12 & -0.538552 & -0.520488 & -0.515930 & -0.53693 \\
\hline 14 & -0.538552 & -0.520488 & -0.515930 & -0.536935 \\
\hline 16 & -0.538552 & -0.520488 & -0.515930 & -0.536935 \\
\hline 18 & -0.538552 & -0.520488 & -0.515930 & -0.536935 \\
\hline 20 & -0.538552 & -0.520488 & -0.515930 & -0.536935 \\
\hline Keller-box & -0.538552 & -0.520488 & -0.515930 & -0.536935 \\
\hline
\end{tabular}

Table $6 s(+\infty, \xi)$ at different values of $\xi$ when $\hbar=-\mathbf{1}$

\begin{tabular}{|c|c|c|c|c|}
\hline iter. & $\xi=0.2$ & $\xi=0.4$ & $\xi=0.6$ & $\xi=0.8$ \\
\hline 4 & -0.078289 & -0.185733 & -0.332014 & -0.472247 \\
\hline 6 & -0.078294 & -0.186013 & -0.337691 & -0.581415 \\
\hline 8 & -0.078294 & 0.186003 & -0.337040 & -0.542247 \\
\hline 12 & -0.078294 & -0.186003 & -0.337103 & -0.551260 \\
\hline 16 & -0.078294 & -0.186003 & -0.337103 & -0.552297 \\
\hline
\end{tabular}




\begin{tabular}{|c|c|c|c|c|}
\hline 18 & -0.078294 & -0.186003 & -0.337103 & -0.552434 \\
\hline 20 & -0.078294 & -0.186003 & -0.337103 & -0.552397 \\
\hline 22 & -0.078294 & -0.186003 & -0.337103 & -0.552406 \\
\hline 26 & -0.078294 & -0.186003 & -0.337103 & -0.552405 \\
\hline Keller-box & -0.078294 & -0.186003 & -0.337103 & -0.552406 \\
\hline
\end{tabular}

\section{CONCLUSION}

The purpose of the current study was to determine the applicability and efficiency of the spectral homotopy analysis method (SHAM) in solving nonlinear partial differential equations. The numerical investigation was carried out using the classic unsteady von Kármán equations. Numerical solutions of the radial and tangential skin friction coefficients were computed and validated against results present in the literature and using the Kellerbox numerical scheme. The comparison revealed matching results for a certain level of accuracy. Computational efficiency of the SHAM was measured in terms of the number of iterations taken to give converging results. Convergence rates were relatively very fast, that is few iterations were required to give converging results. This study has shown the potential of the SHAM in solving nonlinear partial differential equations. The current success of the method adds to a growing body of literature on the development of improved accurate and efficient tools for solving nonlinear equations. The current study has only examined a nonlinear system of equations without flow parameters. Further work needs to be done to establish whether the method generally works for the common types of nonlinear partial differential equations encountered specifically in fluid flow analysis, including larger systems, strong nonlinearity and large parameter values in the nonlinear equations to mention a few.

\section{ACKNOWLEDGEMENTS}

This work is based on the research supported in part by the National Research Foundation of South Africa (Grant No: 85596).

\section{REFERENCES}

Abdou, M. (2010). New analytic solution of von Kármán swirling viscous flow. Acta Appl. Math. 111, 7-13.

Attia, H. A. (2009). Steady flow over a rotating disk in porous medium with heat transfer. Nonlinear Analysis: Modelling and Control 14(1), 21-26.

Bouffanais, R. and D. L. Jacono (2009). Transitional cylindrical swirling flow in presence of a flat free surface. Computers \& Fluids 38, 1651-1673.

Canuto, C., M. Hussaini, A. Quarteroni, and T. Zang (1988). Spectral Methods in Fluid Dynamics. Berlin, Germany.: Springer-Verlag.
Canuto, C., M. Y. Hussaini, A. Quarteroni, and T. A.Zang (2007). Spectral methods: Evolution to complex geometries and applications to fluid dynamics. Berlin, Germany.: Springer-Verlag.

Cebeci, T. and P. Bradshaw (1984). Physical and Computational Aspects of Convective Heat Transfer. New York.: Springer.

Chawla, S., P. Srivastava, and A. Gupta (2006). Spin-down of the von Kármán flow. International Journal of Non-Linear Mechanics 41, 426-431.

Cochran, W. (1934). The flow due to a rotating disc. Proc. Cambridge Phil. Soc. 30, 365 -375.

Dernoncourt, B., J.-F. Pinton, and S. Fauve (1998). Experimental study of vorticity filaments in a turbulent swirling flow. Physica D 117, 181190.

Devi, S. and R. U. Devi (2011). On hydromagnetic flow due to a rotating disk with radiation effects. Nonlinear Analysis: Modelling and Control 16(1), 17-29.

Dong, Q., S. Santhanagopalan, and R. White (2008). Comparison of numerical solutions for the fluid motion generated by a rotating disk electrode. ournal of The Electrochemical Society 155(9), B963-B968.

El-Nahhas, A. (2007). Analytic approximations for von Kármán swirling flow. Proc. Pakistan Acad. Sci. 44(3), 181-187.

Hesthaven, J., S. Gottlieb, and D. Gottlieb (2007). Spectral methods for time-dependent problems. United Kingdom.: Cambridge University Press.

Liao, S. (2003). Beyond Perturbation: Introduction to the Homotopy Analysis Method. Boca Raton.: Chapman \& Hall/ CRC Press.

Liao, S. (2012). Homotopy Analysis Method in Nonlinear Differential Equations. Berlin Heidelberg.: Springer.

Makukula, Z., S. Motsa, and P. Sibanda (2012). On a linearisation method for Reiner-Rivlin swirling flow. Journal of Computational and Applied Mathematics 31(1), 95-125.

Makukula, Z., P. Sibanda, and S. Motsa (2010a). A note on the solution of the von Kármán equations using series and Chebyshev spectral methods. Boundary Value Problems, Volume 2010, Article ID 471793,doi:10.1155/2010/471793, 17 pages.

Makukula, Z., P. Sibanda, and S. Motsa (2012).On new solutions for heat transfer in a viscoelastic fluid between parallel plates. International Journal of Mathematical Models 
and Methods in Applied Sciences 4(4), 221230 .

Makukula, Z., P. Sibanda, and S. S. Motsa (2010b). A novel numerical technique for twodimensional laminar flow between two moving porous walls. Mathematical Problems in Engineering Volume 2010, Article ID 528956, doi:10.1155/2010/528956,15 pages.

Molla, M., S. Saha, and M. Hossain (2012). The effect of temperature dependent viscosity on mhd natural convection flow from an isothermal sphere. Journal of Applied Fluid Mechanics 5(2), 25-31.

Motsa, S. (2013). On the practical use of the spectral homotopy analysis method and local linearisation method for unsteady boundary layer flows caused by an impulsively stretching plate. Numerical Algorithms. http://dx.doi.org/10.1007/s11075-013-9766-z

Motsa, S., P. Sibanda, and S. Shateyi (2010). A new spectral homotopy analysis method for solving a nonlinear second order BVP. Commun. Nonlinear Sci. Numer. Simulat. 15 2293-2302.

Nayagam, V., R. Balasubramaniam, and F. Williams (2009). Diffusion flames over a melting polymer disk in von Kármán swirling flows. Combustion and Flame 156, 16981704.

Öztekin, A., B. Seymour, and E. Varley (2002). Unsteady stratified swirling shear flows. Mathematical and Computer Modelling 36, 321-337.

Poncet, S., R. Schiestel, and R. Monchaux (2007). Turbulent von Kármán flow between two counter-rotating disks. In Lyon (Ed.), Proceedings of the 8th International Symposium on Experimental and Computational Aerothermodynamics of Internal Flows, pp. Paper reference: ISAIF80013.

Prasad, K., K.Vajravelu, and A. Sujatha (2013). Influence of internal heat generation/absorption, thermal radiation, magnetic field, variable fluid property and viscous dissipation on heat transfer characteristics of a maxwell fluid over a stretching sheet. Journal of Applied Fluid Mechanics 6(2), 249-256.

Prasad, K., K. Vajravelu, P. S. Datti, and B. T. Raju (2013). Mhd flow and heat transfer in a powerlaw liquid film at a porous surface in the presence of thermal radiation. Journal of Applied Fluid Mechanics 6(3), 385-395.
Rashad, A. M., A. J. Chamkha, and M. M. Abdou (2013). Mixed convection flow of nonNewtonian fluid from vertical surface saturated in a porous medium filled with a nanofluid. Journal of Applied Fluid Mechanics 6(2), 301-309.

Rashidi, M., T. Hayat, E. Erfani, S. M. Pour, and A. A-Hendi (2011). Simultaneous effects of partial slip and thermal-diffusion and diffusion-thermo on steady mhd convective flow due to a rotating disk. Communications in Nonlinear Science and Numerical Simulations 16(11), 4303-4317.

Rashidi, M., H. Shahmohamadi, and S. Dinarvand (2008). Analytic approximate solutions for unsteady two-dimensional and axisymmetric squeezing flows between parallel plates. Mathematical Problems in Engineering Volume 2008, Article ID 935095, 13 pages.

Sahoo, B. (2009). Effects of partial slip, viscous dissipation and joule heating on von Kármán flow and heat transfer of an electrically conducting non-Newtonian fluid. Commun. Nonlinear Sci. Numer. Simulat. 14, 29822998.

Sibanda, P., S. Motsa, and Z. Makukula (2012). A spectral homotopy analysi method for heat transfer flow of a third grade fluid between parallel plates. International Journal of Numerical Methods for Heat \& Fluid Flow 22(1), 4-23.

Trefethen, L. (2000). Spectral Methods in MATLAB. Philadelphia.: SIAM.

Turkyilmazoglu, M. (2010). Purely analytic solutions of magnetohydrodynamic swirling boundary layer flow over a porous rotating disk. Computers \& Fluids 39, 793-799.

Turkyilmazoglu, M. (2011). Exact solutions for the incompressible viscous fluid of a rotating disk flow. Progress in Applied Mathematics 1(1), 90-97.

Urzay, J., V. Nayagam, and F. Williams (2011). Theory of the propagation dynamics of spiral edges of diffusion flames in von Kármán swirling flows. Combustion and Flame 158, 255-272.

von Kàrmàn, T. (1921). Uberlaminare und turbulence reibung. ZAMM 52(1), 233-252.

$\mathrm{Xu}, \mathrm{H}$. and S. Liao (2006). A series solution of the uunsteady von Kármán swirling viscous flows. Acta. Appl. Math. 94, 215-231.

Zandbergen, P. and D. Dijkstra (1987). von Kármán swirling flows. Ann. Rev. Fluid Mech. 19,46-497. 